\title{
Formation of a large scale spot-like structure of the total deposition due to a powerful elevated finite source
}

\author{
O. Y. Skrynyk ${ }^{1}$, R. I. Chernysh ${ }^{1}$, and Y. Y. Hrytsyuk ${ }^{2}$ \\ ${ }^{1}$ Ukrainian Research Hydrometeorological Institute, Kyiv, Ukraine \\ ${ }^{2}$ Ternopil National Pedagogical University, Ternopil, Ukraine
}

Received: 4 November 2009 - Revised: 13 February 2010 - Accepted: 30 March 2010 - Published: 14 April 2010

\begin{abstract}
The process of formation of a large scale spot-like structure of the cumulative deposition pattern due to a powerful elevated finite lifetime source is studied using numerical solutions of a model diffusion problem. A possible cause of spottiness at regional scale, namely the diurnal cycle of the vertical turbulent mixing intensity in the atmospheric boundary layer, is investigated. It is shown that the atmospheric boundary layer diurnal cycle can cause the appearance of the spot-like structure when the source lifetime, $t_{*}$, is much shorter than the diurnal period, $T$.
\end{abstract}

\section{Introduction}

A cumulative deposition pattern, which takes place under intense atmospheric emissions, has very distinct spot-like structure on different spatial scales. In the other words, a field of deposited materials is self-similar (multi-fractal distribution of deposited materials) (Randle et al., 2003).

The spot-like pollution structure at the regional scale (with linear size of $10^{5} \div 10^{6} \mathrm{~m}$ or more) can be observed particularly well on a map that shows the distribution of the major pollutant, ${ }^{137} \mathrm{Cs}$, on the territory of Ukraine after Chornobyl accident (see Fig. 1b). For comparison, in Fig. 1 we also show a map of ${ }^{137} \mathrm{Cs}$ distribution before the accident. Hereinafter by using the term spot-like structure at the regional scale we denote an existence of several local maxima in total deposition.

It is believed that the main reason for the formation of spots at regional scales is the convective cloud activity, i.e. "damp" aerosol scavenging with atmospheric precipitation ("washout") (see e.g. Clark and Smith, 1988; Puhakka, 1990). However precipitation was not observed when the southern radioactive trace was forming on the territory of Ukraine (Sedunov, 1989, Fig. 1). Taking into account the above mentioned fact we have hypothesized that along with other factors (precipitation, influence of the relief, surface inhomogeneity, etc.) a periodic change (e.g. diurnal cycle) of the vertical turbulent mixing intensity in the ABL can be a reason causing the formation of a large scale spot-like deposition structure. Let us note that the importance of the diurnal variation of meteorological conditions in the process of formation of the earth surface pollution has been pointed out in many researches (see e.g. Baklanov and Sørensen, 2001; Brandt et al., 2002). However, the effect of the vertical turbulent mixing intensity in the ABL has not been studied in detail.

In our opinion, the hypothesis is quite reasonable and physically clear when the pollution is caused by an instantaneous elevated release (e.g. explosion, with subsequent transport and diffusion of the contamination cloud). For such a case the hypothesis has been confirmed by Voloshchuk et al. (2007). At the same time it is not clear whether the spottiness can be formed when the source is active for a limited time interval, as it occurred during the Chornobyl accident, and under what conditions this can happen.

It should be emphasized that the present work is not an attempt to reconstruct the deposition pattern that took place after the Chornobyl accident. The purpose of this work is to study the mentioned effect in the atmospheric diffusion using known and well approved methods, namely the K-theory. A simple but realistic schemes of parameterization of vertical structure and time evolution of the ABL have been used in the model diffusion problem. Note that the evolution of the diffusion processes considered in this work take place over space and time scales, where the use of the K-theory is justified.

Correspondence to: O. Y. Skrynyk

(skrynyk@univ.kiev.ua) 
(a)

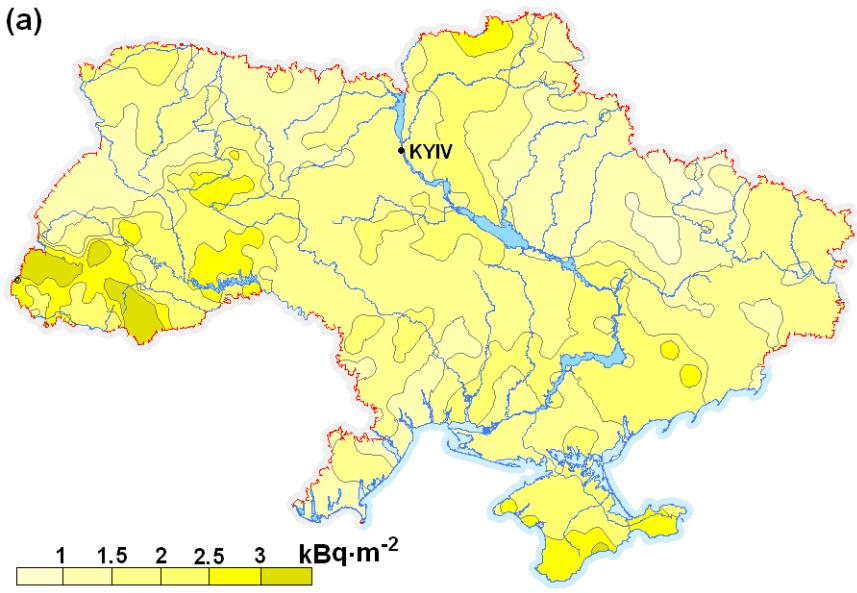

(b)

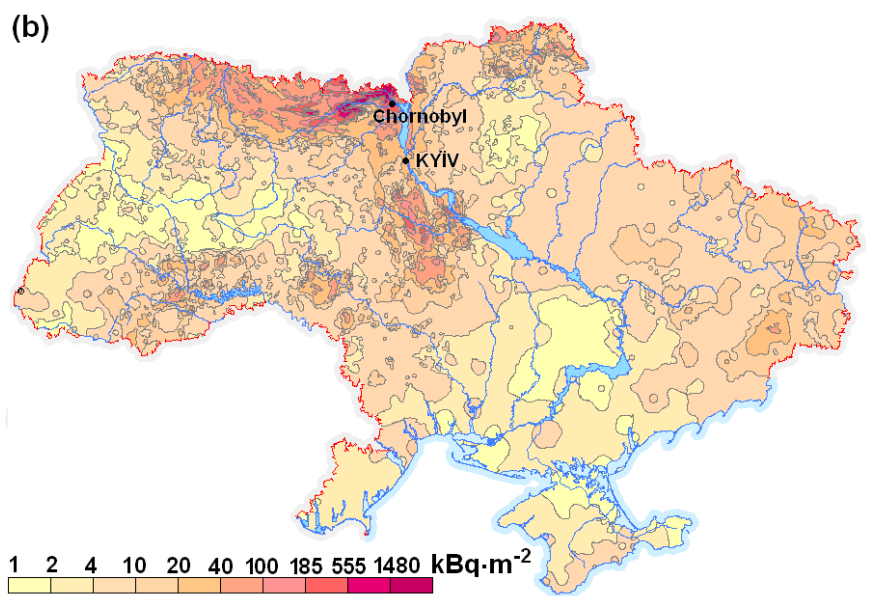

Figure 1. Maps of radioactive contamination of Ukraine by Cesium-137: (a) before-accident pollution (July 1985); (b) pollution after the Chornobyl accident (January 1998) (from Atlas of radioactive contamination of Ukraine (electronic version), 2002).

\section{Parameterization of the atmospheric boundary layer}

For the intensity of the vertical turbulent mixing, i.e. the value of the vertical eddy diffusivity, $K_{z}$, we will use the parameterization that was suggested in paper Mangia et al. (2002). In this parameterization the dependence of diffusivity on stability of ABL is introduced by means of the Obukhov-Monin length scale, $L$.

If $L<0$

$K_{z}=K_{z m}+K_{z c}, K_{z m}=0.3 z u_{*}(1-z / h), K_{z c}=\frac{0.24 z(z / h)^{1 / 3}}{q_{w c}^{4 / 3}} w_{*}$,

$w_{*}=u_{*}\left(-\frac{h}{\kappa L}\right)^{1 / 3}$,

$q_{w c}=\left\{\begin{array}{cl}0.48, & z \leq 0.1 h \\ 1.6 z / h\left(1-e^{-4 z / h}-3 \times 10^{-4} e^{8 z / h}\right)^{-1}, & 0.1 h \leq z \leq h\end{array}\right.$

where $K_{z \mathrm{~m}}$ is the mechanical part of the vertical diffusivity, $K_{z c}$ is the convective part of the vertical diffusivity, $h$ is the convective boundary layer height, $w_{*}$ is the convective velocity scale, $u_{*}$ is the friction velocity, $\kappa$ is the von Kármán constant, $q_{w c}$ is the stability function.

If $L>0$

$K_{z}=\frac{0.3 z u_{*}(1-z / h)}{1+3.7 z / \Lambda}, \quad \Lambda=L(1-z / h)^{\left(1.5 \alpha_{1}-\alpha_{2}\right)}$

where $\alpha_{1}$ and $\alpha_{2}$ are empiric constants $\left(\alpha_{1}=2, \alpha_{2}=3\right.$, Minnesota values).

The parameterization (1)-(2) belongs to a class of parabolic profiles which reproduce a real vertical profile of $K_{z}$, both in a day, and in a night ABL (Nieuwstadt and Van Dop, 1981).
The wind speed profile in the surface layer has been parameterized following the similarity theory of MoninObukhov (Monin and Yaglom, 1971)

$u=\frac{u_{*}}{\kappa}\left[\ln \left(z / z_{0}\right)-\psi_{m}(z / L)+\psi_{m}\left(z_{0} / L\right)\right]$,

where $\psi_{m}(z / L)$ is the stability function. It represents the correction to the logarithmic profile due to non-neutral conditions ( $\psi_{m}=0$ in neutral conditions). The stability function is parameterized using the following relationships (Businger et al., 1971)

$\psi_{m}=\left\{\begin{array}{cc}2 \ln \left(\frac{1+\phi_{m}^{-1}}{2}\right)+\ln \left(\frac{1+\phi_{m}^{-2}}{2}\right)-2 \tan ^{-1}\left(\phi_{m}^{-1}\right)+\frac{\pi}{2}, & L<0 \\ -4.7 z / L, & L>0\end{array}\right.$

$\phi_{m}= \begin{cases}(1-16 z / L)^{-1 / 4}, & L<0 \\ 1+4.7 z / L, & L>0\end{cases}$

where $\phi_{m}$ is dimensionless wind profile.

We assume that above the surface layer the wind speed is constant: $u(z)=u\left(z_{b}\right)$, if $z>z_{b}$, where $z_{b}$ is the surface layer height $\left(z_{b} \approx 5 \div 10 \%\right.$ of the ABL height).

The parameterisations (1)-(2), (3) give continuous values for $K_{z}$ and $u$ at all elevations and all stability conditions from unstable to stable, allowing us to treat dispersion in full diurnal cycle. As a rule, the unstable state prevails in the day time and the stable one at night (Stull, 1988).

To model diffusive processes over some days it is necessary to specify a diurnal cycle of stability. We will introduce the dependence of the Obukhov-Monin length scale on time as follows:

$L^{-1}=B \cos \left(\frac{2 \pi t}{T}+\omega_{0}\right)$,

where $B$ is the amplitude of the diurnal change of $L^{-1}$ (may depend on both the geographical coordinates and the time 
of the year), $T=86400 \mathrm{~s}$ is the full period, $\omega_{0}$ is the initial phase, $t$ is the time of the diffusion.

It should be emphasized that parameterization (4) is only the first approximation, which only qualitatively recreates the real diurnal change of the Obukhov-Monin length. Nevertheless, for the purpose of the present work, parameterization (4) can be considered satisfactory.

The parameterization (1)-(2) is 3-parametrical $\left(K_{z}=\right.$ $\left.f\left(L, h, u_{*}\right)\right)$. Parameters $h$ and $u_{*}$ also undergo a diurnal change. However, in our study we constrained ourselves by taking into account only the daily change of $L$, i.e. we assumed that $h$ and $u_{*}$ are constant (e.g. diurnal mean values can be used). The assumption of constant $u_{*}$ is not very limiting, while the assumption of a constant $h$ is a strong one and that has to be explained. At night $h$ becomes small, the maximum value of $K_{z}$ and its vertical position decrease. However at each elevation the day time value of $K_{z}$ is larger than its night value. We believe that such behaviour of $h$ and $K_{z}$ would not change the results of our study qualitatively. Moreover, we believe that the diurnal change of $h$ will intensify the spottiness effect (the spottiness will appear more distinctly). Because at night some amount of contamination, which happened to be above the height of the night ABL, will simply be transported and will not disperse.

\section{Model diffusion problem}

Let us consider a dispersion problem where the turbulent diffusion of gaseous and aerosol pollutants in the bottom part of the atmosphere is modeled. Assuming that the source of the pollutants is point-like and the pollutants are nonsettling, and orienting $\mathrm{x}$-axis of the coordinate system in the direction of mean wind flow we obtain the following mathematical formulation of the problem:

$\frac{\partial c}{\partial t}+u \frac{\partial c}{\partial x}=K_{y} \frac{\partial^{2} c}{\partial y^{2}}+\frac{\partial}{\partial z} K_{z} \frac{\partial c}{\partial z}$,

$c=c(t, x, y, z), \quad t>0, x>0,|y|<\infty, z_{0}<z<h$,

$K_{y}=$ const.

The initial condition is

$\left.c\right|_{t=0}=0$.

The boundary conditions are

$\left.c u\right|_{x=0}=Q H(t) H\left(t_{*}-t\right) \delta(y) \delta\left(z-h_{*}\right)$,

$Q=$ const., $\quad z_{0}<h_{*}<h$

$\left.c\right|_{|y| \rightarrow \infty} \rightarrow 0$,

$\left.c\right|_{z=z_{0}}=0,\left(z_{0} \neq 0\right)$

$\left.K_{z} \frac{\partial c}{\partial z}\right|_{z=h}=0$.
In expressions (5)-(11) we used the following notations: $c$ is the concentration of pollutants; $t, x, y, z$ are the time and space coordinates respectively; $K_{y}$ is the crosswind eddy diffusivity; $Q$ and $h_{*}$ are the source strength and height; $t_{*}$ is the source lifetime; $\delta(y)$ is the Dirac delta function; $H(t)$ is the Heavyside step function, defined as

$H(t)=\left\{\begin{array}{ll}1, & t>0 \\ 0, & t \leq 0\end{array}\right.$.

In Eq. (5) the wind speed, $u$, and the vertical eddy diffusivity, $K_{z}$, are defined by expressions (3) and (1)-(2) under the condition (4) respectively.

We assumed that $K_{y}$ does not undergo a diurnal change ( $K_{y}=$ const.). The assumption is legitimate because it is known that the major contribution to the crosswind eddy diffusivity is made by the vertical wind shear. We also neglected the diffusion in the direction of the regular transport of pollutants $\left(K_{x}=0\right.$, where $K_{x}$ is the eddy diffusivities along $x$ direction), which allowed us to represent the source through the boundary condition (8). Such a trick is often used in modeling the turbulent diffusion in the presence of a continuous (or finite) source.

The upper boundary condition (11) along the vertical coordinate means that the transport of pollutants into the upper layers is absolutely forbidden. This does not represent a serious limitation for diffusion problems. Such a condition leads to somewhat overestimated intensities of the turbulent contaminant fluxes to the ground. As the lower boundary condition we use the condition of full absorption. Here, however, we do face a problem. The parabolic approximation of the vertical turbulent diffusivity (1)-(2) yields complete forbidding of the turbulent fluxes at $z=0$. From purely mathematical standpoint it means that the boundedness condition is the only correct condition under such a parameterization. In other words, the parabolic parameterization of $K_{z}$ is not compatible with the boundary condition of full (or partial) absorption. To resolve this problem we will lift the point where the lower boundary condition is given. The roughness height, $z_{0}$, can be used as a suitable value for this purpose (10).

Having obtained the solution of problem (5)-(11), (1)-(4) it is easy to find the total deposition to the ground, $c^{*}(x, y)$, using the following formula:

$c^{*}(x, y)=\left.\int_{0}^{\infty} K_{z} \frac{\partial c}{\partial z}\right|_{z=z_{0}} d t$.

Here $c^{*}(x, y)$ is the principal unknown, which needs to be found.

It is practically impossible to obtain the analytic solutions of problem (5)-(11), (1)-(4). Thus, we must carry out a numerical simulation. For this purpose we used a finite-difference method. Using the method of sequential 


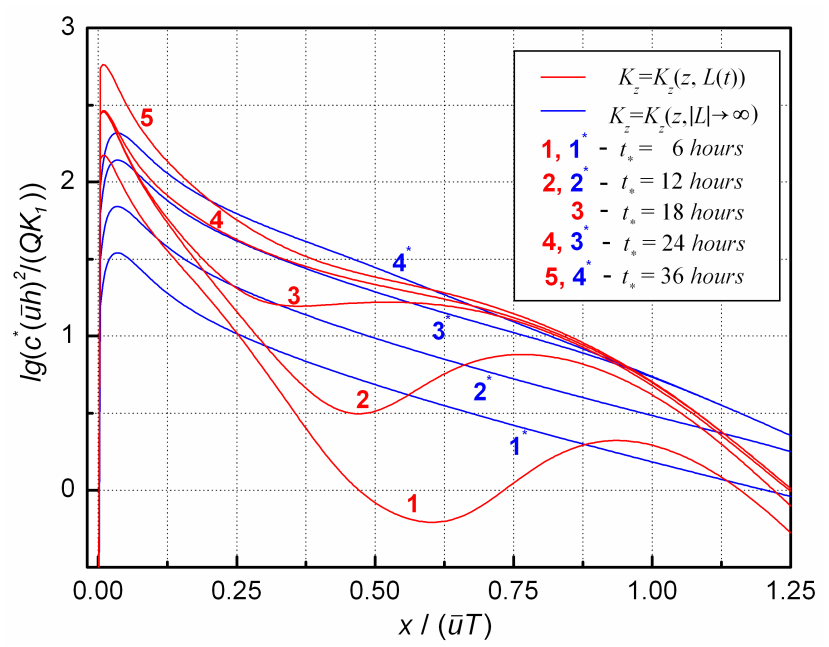

Figure 2. Horizontal profiles (at $y=0$ ) of the normalized total deposition.

component-wise splitting (Marchuk, 1988) we obtained onedimensional problems, to solve which we used the CrankNicholson method (Hoffman, 2001).

\section{The analysis of the results - an empirical criterion for the appearance of a spot-like pollution struc- ture}

The results of the calculations of the total deposition based on the numerical simulations of diffusion problem considered above are shown in Fig. 2. There we present horizontal profiles of the total deposition $c^{*}(x, y)$ at $y=0$ for several values of the source lifetime. The total deposition was normalized by $Q K_{1} /\left(\bar{u}^{2} h^{2}\right)$ where $\bar{u}=\frac{\left.u\left(z_{b}\right)\right|_{L^{-1}=B}+\left.u\left(z_{b}\right)\right|_{L^{-1}=-B}}{2}, K_{1}=$ $\left.\frac{1}{h} \int_{0}^{h} K_{z}\right|_{L^{-1}=0} d z$, and is given in the logarithmic scale. The longitudinal coordinate $x$ is normalized by the distance, which a unit volume of air passes for a period $T=24 \mathrm{~h}$ due to the regular transport $(\bar{u} T)$.

The results of the total deposition calculations when a diurnal change of stability was neglected in the diffusion problem (i.e. when $|L| \rightarrow \infty$, that corresponds to neutral conditions) are placed in Fig. 2 as well. The latter has been done in order to show that the occurrence of more than one maxima of the total deposition is due to the periodic change of $K_{z}$ only.

In all of the cases the actual values of the input parameters for the diffusion problem were kept the same. To obtain numerical solutions of diffusion problem we used the following values of parameters: $B=0.01 \mathrm{~m}^{-1}$, according to paper Irwin (1979) it means that the stability in the ABL changes from Pasquill-Gifford stability class $\mathrm{C}$ to stability class $\mathrm{E} ; \omega_{0}=\frac{\pi}{2}$, it corresponds to a situation when releases begin approxi- mately at morning time; $z_{0}=0.1 \mathrm{~m} ; z_{b}=50 \mathrm{~m} ; h_{*}=500 \mathrm{~m}$; $h=1000 \mathrm{~m} ; Q=1 \mathrm{~kg} \mathrm{~s}^{-1} ; u_{*}=0.35 \mathrm{~m} \mathrm{~s}^{-1} ; \bar{u}=6.12 \mathrm{~m} \mathrm{~s}^{-1}$; $K_{y}=300 \mathrm{~m}^{2} \mathrm{~s}^{-1} ; K_{1}=17.5 \mathrm{~m}^{2} \mathrm{~s}^{-1}$.

Based on the analysis of the obtained results we reached the conclusion that the effect of spottiness takes place not only in the presence of an instantaneous source, but also in the presence of a source with a finite lifetime. The spotlike pollution structure of the cumulative deposition can be clearly seen in Fig. 2 at relatively small values of $t_{*}$ (function $c^{*}(x, 0)$ has more than one maximum). When the lifetime of the source gets longer, the accumulation of the pollutants at the local minima of $c^{*}(x, 0)$ becomes more intensive. This leads to some smoothing of the pollutant field. As $t_{*}$ increases, all local maxima, except for the first one, disappear and function $c^{*}(x, 0)$ becomes monotonically decreasing in the interval $x>x_{\max 1}$, where $x_{\max 1}$ is the position of the first maximum. At $t_{*}=T, 2 T, 3 T \ldots$ the function becomes strictly monotone, while at $t_{*} \in(T i, T(i+1))$, where $i \in N$, the strict monotony might not hold. It is difficult to explain such behavior of the total deposition. A possible reason is following: when the lifetime of the source gets longer, every location ( $x$ points) experiences different $K_{z}$ value due to the different stability conditions ( $L$ value) varying during the daily cycle, while for very short puffs only a limited range of $L$ values are "seen" when the puff travels over a specific location.

The existence of a certain criterion for the spottiness can be justified relatively easy. On the one hand, it is obvious that the periodic change of stability in the ABL will result in several local maxima of total concentration deposition when the instantaneous source acts. Qualitatively this process can be pictured as follows: the wind transports the cloud of contaminants and during this transportation the vertical turbulent fluxes of contaminants to the ground are "switched on" in the daytime and "switched off" at night. On the other hand, it is also obvious that the spottiness will not appear when $t_{*} \rightarrow \infty$ because of the effect of averaging of many puffs that form the plume. Thus, a finite source lifetime for which the spot-like pollution structure vanishes has to exist.

We carried out numeric experiments with different values of input model parameters, like $B$ and $\omega_{0}\left(\omega_{0} \in[0 ; 2 \pi]\right.$, $B \in[0.005 ; 0.05])$. The value of these parameters influences the maxima of the total deposition and their positions ( $x$ coordinates). But under any value of $B$ and $\omega_{0}$ the spot-like structure was observed.

The conclusions presented above are obtained based on the results of numerous computer simulations, i.e. they are empirical. It is very hard to prove them in a rigorous mathematical sense.

From the analysis of the results of the numerical simulations it follows that the effect of spottiness takes place when condition $t_{*} / T<1$ is satisfied. This relation can be considered as an empirically established criterion for the appearance of a spot-like pollution structure. 


\section{Conclusions}

Empirical data and experiments, which can help to understand the effect of diurnal change in the vertical turbulent mixing intensity on the surface contamination pattern, are very scarce. With the exception of deposition patterns generated by Chornobyl accident release, we do not know of any other relevant experimental data. Thus, at present mathematical modelling (numerical simulation) remains the only way to verify the hypothesis that spottiness may be caused by the diurnal cycle of the vertical turbulent mixing intensity in the ABL.

In this work we have studied the process of formation of the total dry deposition to the ground in the presence of a finite lifetime point source. From the analysis of the results it follows that the effect of large scale spottiness due to the diurnal cycle of the vertical turbulent mixing intensity in the ABL takes place when certain conditions are satisfied. We have formulated an empirical criterion of the appearance of a spot-like pollution structure, which is determined by the ratio of the source lifetime and the characteristic time of the periodic changes in the intensity of the vertical turbulent mixing.

Acknowledgement. Authors wish to thank anonymous reviewers for valuable comments and suggestions.

Edited by: D. Giaiotti

Reviewed by: two anonymous referees

\section{References}

Baklanov, A. and Sørensen, J. H.: Parameterisation of radionuclide deposition in atmospheric long-range transport modelling, Phys. Chem. Earth (B), 26(10), 787-799, 2001.

Brandt, J., Christensen, J. H., and Frohn, L. M.: Modelling transport and deposition of caesium and iodine from the Chernobyl accident using the DREAM model, Atmos. Chem. Phys., 2, 397-417, 2002, http://www.atmos-chem-phys.net/2/397/2002/.

Businger, J. A., Wyngaard, J. C., Izumi, Y., and Bradley, E. F.: Fluxprofile relationship in the atmospheric surface layer, J. Atmos. Sci., 28, 181-189, 1971.
Clark, M. J. and Smith, F. B.: Wet and dry deposition of Chernobyl releases, Nature, 332, 245-249, 1988.

Atlas of radioactive contamination of Ukraine (electronic version), Kyiv, Ministry on Emergency Situations of Ukraine, 2002.

Irwin, J. S.: A theoretical variation of the wind profile power-law exponent as a function of surface roughness and stability, Atmos. Environ., 13, 191-194, 1979.

Hoffman, J. D.: Numerical methods for engineers and scientists, Basel, New York, 2001.

Mangia, C., Moreira, D. M., Schipa, I., Degrazia, G. A., Tirabassi, T., and Rizza, U.: Evaluation of a new eddy diffusivity parameterisation from turbulent Eulerian spectra in different stability conditions, Atmos. Environ., 36, 67-76, 2002.

Marchuk, G. I.: Metody raschepleniya (Methods of splitting), Nauka, Moscow, 1988.

Monin, A. S. and Yaglom, A. M.: Statistical fluid mechanics, MIT Press, Cambridge, 1971.

Nieuwstadt, F. T. M. and Van Dop, H. (Eds.): Atmospheric turbulence and air pollution modeling. A course held in the Hague, 21-25 September, Reidel Publishing Company, 1981.

Puhakka, T.: Meteorological factor influencing the radioactive deposition in Finland after the Chernobyl accident, J. Appl. Meteor., 29, 813-829, 1990.

Randle, K., Sokhi, R. S., and Cooper, J. R.: Radioactive Releases in the Environment: Impact And Assessment, John Wiley \& Sons, Chichester, 473 pp., 2003.

Sedunov, Yu. S., Borzilov, V. A., Klepikova, N. V., Chernokozhin, E. V., and Troyanova, N. I.: Physicomathematical modeling of the regional transport of radioactive pollutants in the atmosphere in consequence of the Chernobyl accident, Russ. Meteorol. Gidrol., 9, 5-10, 1989.

Stull, R. B.: An introduction to boundary layer meteorology, Kluwer Academic Publishers, Dordrecht, 666 pp., 1988.

Voloshchuk, V. M., Skrynyk, O. Y., and Hrytsyuk, Y. Y.: Formation mechanism of a large-scale spot-like pollution structure of the underlying surface under intense aerosol injections into the atmospheric boundary layer, Reports of the National Academy of Sciences of Ukraine, 4, 115-120, 2007. 